\title{
Water Saturation Estimation Using Crossplot Method Based on Turgut- Yamamoto Equation
}

\author{
Sismanto ${ }^{1}$, Rah Ajeng Putri Tresnani ${ }^{2}$ \\ ${ }^{1}$ Geophysics Laboratory, Physics Department, \\ Mathematical and Natural Science Faculty, Gadjah Mada University. Yogyakarta, Indonesia \\ ${ }^{2}$ SpectrumGeo - Jakarta, Indonesia \\ 1e-mail: sismanto@ugm.ac.id \\ ${ }^{2}$ e-mail: ajeng.putri@spectrum-jakarta.com
}

\begin{abstract}
Hydrocarbon reservoirs are characterized by reservoir parameters such as porosity, permeability, and water saturation. There is a close relationship among wave parameters, elastic parameters, and rock properties, and ultimate reservoir parameters have been established in continuum mechanics and rock physics. Therefore, one should be able to determine the reservoir parameters, especially water saturation from seismic waveforms. We used Biot's equation of elastic wave propagation in porous and fluid saturated medium. The approximations of Turgut-Yamamoto had simplified Biot's formula to obtain the relationship between attenuation and water saturation and then make it in linear form, thus it can be used as standard curves. The linearization Turgut-Yamamoto standard curve is more obvious to the dependency of attenuation to water saturation. To test the method, we used a log data from "BARRETTA" Well in North Sumatera Basin. Total thick of the prospect zone on KTP Formation is about $72 \mathrm{ft}$. The error of water saturation estimation by the cross-plot method in standard curves to log analysis on the prospect zone of KTP Formation is about $3 \%$.
\end{abstract}

Keywords: Water saturation estimation, attenuation, Turgut-Yamamoto.

\section{INTRODUCTION}

The characteristics of a hydrocarbon i.e. compressibility, porosity, permeability and water saturation are very important information. The seismic technology, the exploration geophysical methods, that has been developed for the lithology and reservoir parameters determination. Generally, theory, field data, and experiments have been able to be used to prove that there is a close relationship among waves, elastics and reservoir parameters. Many studies have been conducted both in theoretical analysis [1] and in experimental analysis [2] to prove that there are a strong relationship among the petrophysical reservoir parameter, inelastic and elastic seismic wave parameter such as attenuation coefficient, quality factor, amplitude (coefficient reflection), frequency, and velocity [3]. It shows that these petrophysical parameters essentially are the other form of the rock elasticity value, and it provides a direct influence on the shape and behavior of seismic waveform recorded on the surface. Consequently, it should be possible to determine the reservoir parameters, especially water saturation, from the seismic waveform. The problem is how to extract it.

Petrophysical parameters of the reservoir have the significant effect on the characteristics and shape of seismic waves. The conventional methods to obtain reservoir parameters are core and log data analysis. Log data is used to identify productive zone, to determine depth and thickness of the zone, to distinguish water, oil, or gas within a reservoir, and to estimate hydrocarbon reserves [4]. Log data interpretation is a process to convert rock physical parameters of a formation (resistivity, density, interval transit time, etc.) to the petrophysical parameter (porosity, permeability, hydrocarbon saturation, etc).

The attenuation of compressional and shear waves in rocks depends on the physical state and saturation conditions. Generally, attenuation varies much more than the seismic velocities as a result of changes in the physical state of the material. Seismic attenuation can also be expressed in terms of the quality factor $(Q)$, which is proportional to the inverse of the loss of energy per wavelength. The larger the quality factor, the better the rock to transmit seismic energy [5]. In general, the attenuation increases with increasing liquid concentration.

The study of numerical models to visualize the behavior of the seismic wave propagation in fluid filled porous media have been studied thoroughly to understand the influence of the petrophysical reservoir parameters such as permeability with velocity, frequency, and wave amplitude by Turgut and Yamamoto (1988). Based on this comprehension, it evokes a query regarding the calculation method to determine the petrophysical reservoir parameter which in particular is permeability, from seismic waveform recorded on the surface [6]. 
In oil and gas exploration, the quantity of water saturation is commonly obtained from conventional petrophysical calculation analysis. This study attempts to find the different method to find water saturation, besides coring. The significant aspect of this study is finding out the relationship between water saturation and attenuation. The procedural work is connecting seismic wave information to reservoir parameters based on log data analysis. The method used to make standard curves based on Biot's equation which then had been simplified by Turgut-Yamamoto, and make the equation to be a linear form [7].

\section{METODOLGY}

Two basic equations describing the relationship between dilatational and shear waves in fluid-saturated porous (unconsolidated) isotropic and elastic media such as marine sediments are according to Biot [8] and [9];

$$
\begin{aligned}
& \mu \nabla^{2} \vec{u}+(\bar{H}-\mu) \nabla \theta-C \nabla \zeta_{p}=\rho \frac{\partial^{2} \vec{u}}{\partial t^{2}}-\rho_{f} \frac{\partial^{2} \vec{V}}{\partial t^{2}} \\
& C \nabla \theta-M \nabla \zeta_{p}=\rho_{f} \frac{\partial^{2} u}{\partial t^{2}}-m \frac{\partial^{2} \vec{V}}{\partial t^{2}}-\frac{\eta}{k_{p}} \frac{\partial \vec{V}}{\partial t}
\end{aligned}
$$

where $\vec{u}$ is the frame displacement vector, $\vec{V}$ is the seepage displacement vector; $\theta=\operatorname{div} \vec{u} \zeta_{p}=\operatorname{div} \overrightarrow{\mathrm{V}} \rho_{r}$ is the grain density, $\rho$ is the bulk density $\left[\rho=(1-\Phi) \rho_{r}+\Phi \rho_{f}\right] \rho_{f}$ is the density of the fluid, $\Phi$ is porosity, $\eta$ is fluid viscosity, and $k_{p}$ is the coefficient of permeability. $\bar{H}, C$, and $M$ are the Biot's elastic modulus, $\mu$ is the shear modulus, $m$ is the virtual mass expressed as $m=\alpha \rho_{f} / \Phi, \alpha=1.25$ [10]. Meanwhile, Biot's elastic modulus can be expressed by the following equation [11];

$$
\begin{aligned}
& \bar{H}=\frac{\left(K_{r}-K_{b}\right)^{2}}{D-K_{b}}+K_{b}+\frac{4}{3} \mu, \\
& C=\frac{K_{r}\left(K_{r}-K_{b}\right)}{D-K_{b}}, \\
& M=\frac{K_{r}^{2}}{D-K_{b}}, \\
& D=K_{r}\left[1+\phi\left[\frac{K_{r}}{K_{f}}-1\right]\right]
\end{aligned}
$$

where $K_{r}$ is the bulk modulus of the grain, $K_{f}$ is the bulk modulus of the fluid in the pores, and $K_{b}$ is the bulk modulus of the skeletal frame. Based on Turgut and Yamamoto [10], the bulk modulus of skeletal frame $K_{b}$ and the porosity $\Phi$ are related to the shear modulus as

$$
\begin{aligned}
& K_{b}=\left(\frac{2 \sigma}{1-2 \sigma}+\frac{2}{3}\right) \mu, \\
& \Phi=\frac{K_{f}\left(K_{r}-K\right)}{\left(K_{r}-K_{f}\right)\left(K-K_{b}\right)}
\end{aligned}
$$

Shear modulus $\mu$, Poisson's ratio $\sigma$, and bulk modulus $K$ can be obtained from the relation of

$$
\begin{aligned}
\mu & =\rho V_{s}^{2}, \\
\sigma & =\frac{3 K-2 \mu}{2(3 K+\mu)}, \\
K & =\rho\left(V_{p}^{2}-\frac{4}{3} V_{s}^{2}\right)
\end{aligned}
$$

Biot's Eq.(1) and Eq. (2) can be derived by Turgut and Yamamoto [10] in the slowness square of P and S waves form, i.e.

$$
\begin{gathered}
\frac{1}{V_{p}^{2}}=\frac{-\left(H q+M \rho-2 C \rho_{f}\right) \pm \sqrt{\left(H q+M \rho-2 C \rho_{f}\right)^{2}-4\left(C^{2}-M H\right)\left(\rho_{f}^{2}-\rho q\right)}}{2(C-M H)}, \text { and } \\
\frac{1}{V_{s}^{2}}=\frac{q \rho-\rho_{f}^{2}}{q \mu}
\end{gathered}
$$


where,

$$
q=\frac{\alpha^{\prime} \rho_{f}}{\phi}-i \frac{\eta F}{\omega k}
$$

$q$ is a complex number, and for low-frequency correction factor, $F$ equals 1 . The real and the imaginary parts of velocity indicate the velocity and the attenuation of wave propagation in the medium. Turgut and Yamamoto [12] solve this equation for unconsolidated rocks such as marine sediments, sandstone, limestone, and porous rock in general that have a high quality factor. From these equations, it was derived the attenuation-frequency relationships. For most marine sediment with high-quality factor $Q$, the intrinsic attenuation $Q^{-1}$ can be showed as [10] equation,

$$
Q^{-1}=\frac{\frac{V_{\infty}^{2}}{V_{0}^{2}}-1}{\frac{q_{i}}{A}+\frac{A}{q_{i}} \cdot \frac{V_{\infty}^{2}}{V_{0}^{2}}}
$$

where $q_{i}(=\eta / k \omega)$ is the imaginary part of $q$, and $A=\left(\rho m-\rho_{f}^{2} / \rho\right) V_{0}$ and $V_{\infty}$ are the zero and high-frequency velocities, such as [10], i.e.,

$$
\begin{gathered}
V_{0}^{2}=\frac{H}{\rho}, \\
\text { and } V_{\infty}^{2}=\frac{H m+M \rho-2 C \rho_{f}}{\rho m-\rho_{f}^{2}}
\end{gathered}
$$

In the experiments, Turgut - Yamamoto uses ultrasonic wave source, so that it is difficult enough to implement in low-frequency, due to the seismic wave having less than $200 \mathrm{~Hz}$. For practical purposes, Sismanto [7] modify and develop the equation to be transformed into a linear form that it is more operational. According to the approximation for marine sedimentary and unconsolidated rocks, Turgut and Yamamoto [12] obtained the attenuation $Q^{-1}$ relationship with frequency $\omega$ as a quadratic function. In the low frequency, Eq.(15) has a linear relationship. It can derive the linear relationship by means of Taylor series in frequency, and cut the power of that is greater than one [13], it yields

$$
Q^{-1} \approx \frac{2 \pi k_{p}}{\eta}\left(\frac{\rho m-\rho_{f}{ }^{2}}{\rho}\right)\left(\frac{V_{\infty}{ }^{2}}{V_{o}^{2}}-1\right) f
$$

Eq.(18) then is used to make further analysis of the relationships between water saturation, which is contained within density and attenuation parameters. For porous rock which contains water and gas, the density may be found as

$$
\rho=(1-\Phi) \rho_{m a}+\Phi\left[S_{w} \rho_{w}+\left(1-S_{w}\right) \rho_{g}\right\rfloor
$$

\section{A. Petrophysical Analysis}

In a petrophysical analysis, there are some equations used to calculate parameter values. The existence of shale on a reservoir may cause an error for estimating porosity and water saturation values. Shale on a formation may decrease resistivity contrast between oil or gas, and water; thus it is being difficult to define the productivity of a zone [14]. Then, Larionov [15] gives an equation to calculate shale volume $\left(V_{s h}\right)$ for unconsolidated rocks, such as sandstone, as

$$
V_{s h}=0,083\left(2^{\left(3.7 \times I_{G R}\right)}-1\right)
$$

But, it may be first to consider gamma ray index $\left(I_{G R}\right)$ value as

$$
I_{G R}=\frac{G R-G R_{\min }}{G R_{\max }-G R_{\min }}
$$

$G R, G R_{\min }$ and $G R_{\max }$ are gamma ray log data in reading, minimum, and maximum respectively.

Porosity value may be found by an equation, which combines porosity values from density $\log \left(\Phi_{N_{C}}\right)$ and neutron log calculation $\left(\Phi_{D c}\right)$, i.e.

$$
\Phi_{A v}=\frac{\Phi_{N c}+\Phi_{D c}}{2}
$$


The passing of a reservoir formation volume (permeability) can be determined from empirical formula of Wyllie and Rose [4], i.e.

$$
K=c \frac{\Phi^{3}}{\left(S_{\text {wirr }}\right)^{2}}
$$

$c$ is constant, besides porosity and permeability, water saturation is also an important parameter in log analysis. By knowing the saturation values of a formation then hydrocarbon volume of a reservoir can also be known. For shaly formation, water saturation $S_{w}$ can be calculated through Simandoux's formula [4], i.e.

$$
S_{w}=\left(\frac{0,4 \times R_{w}}{\Phi^{2}}\right) \times\left[\sqrt{\left(\frac{V_{s h}}{R_{s h}}\right)^{2}+\frac{5 \times \phi^{2}}{R_{t} \times R_{w}}}-\frac{V_{s h}}{R_{s h}}\right] \times 100 \%
$$

where $R_{w}$ is resistivity of water, $R_{s h}$ is resistivity of shale and $R_{t}$ is total resistivity. The density of a rock is defined as the ratio between mass and volume of a rock.

\section{B. Elastic Wave Velocity on Sediment}

The sedimentary porous rocks generally show lower velocities than igneous and metamorphic rocks. The relationship between compressional (P) and shear (S) waves, $V_{P^{-}} V_{S}$, now seems to be useful parameters for rock characterization. Castagna [16] and Mavko [17] include the formulation of $V_{P^{-}} V_{S}$ relationship as polynomial form along with coefficient of each lithology (Table 1) i.e.

$$
V_{s}=a_{2} V_{p}^{2}+a_{1} V_{p}+a_{0}(\mathrm{~km} / \mathrm{s})
$$

Table 1. Polynomial coefficient of $V_{P-} V_{S}$ relationship [17].

\begin{tabular}{lllll}
\hline Lithology & $\boldsymbol{a}_{\mathbf{2}}$ & $\boldsymbol{a}_{\mathbf{1}}$ & $\boldsymbol{a}_{\boldsymbol{o}}$ & $\boldsymbol{V}_{\boldsymbol{p}}(\mathbf{k m} / \mathbf{s})$ \\
\hline sandstone & 0 & 0.80416 & -0.85588 & $2-6$ \\
limestone & -0.05508 & 1.01677 & -1.03049 & $1.5-6$ \\
dolomite & 0 & 0.58321 & -0.07775 & $4.5-6.4$ \\
claystone & 0 & 0.76969 & -0.86735 & $1.5-5$ \\
\hline
\end{tabular}

Castagna, [16] encloses also the relationship between $V_{P}$ and bulk density $\left(\rho_{b}\right)$ as polynomial along with coefficient of each lithology (Table 2), i.e.

$$
\begin{aligned}
\rho_{b} & =a V_{p}^{2}+b V_{p}+c \\
\rho_{b} & =d V_{p}^{f}
\end{aligned}
$$

Table 2. Polynomial coefficient of $V_{P}-\rho_{b}$ relationship [16]

\begin{tabular}{lllllll}
\hline Lithology & $\boldsymbol{a}$ & $\boldsymbol{b}$ & $\boldsymbol{c}$ & $\boldsymbol{d}$ & $\boldsymbol{f}$ & $\boldsymbol{V}_{\boldsymbol{p}}(\mathbf{k m} / \mathbf{s})$ \\
\hline claystone & -0.0261 & 0.373 & 1.458 & 1.75 & 0.265 & $1.5-5$ \\
sandstone & -0.0115 & 0.261 & 1.515 & 1.66 & 0.261 & $1.5-6$ \\
limestone & -0.0296 & 0.461 & 0.963 & 1.50 & 0.225 & $3.5-6.4$ \\
dolomite & -0.0235 & 0.390 & 1.242 & 1.74 & 0.252 & $4.5-7.1$ \\
anhydrite & -0.0203 & 0.321 & 1.732 & 2.19 & 0.160 & $4.6-7.4$ \\
\hline
\end{tabular}

The study of relationship between velocity, frequency and permeability $\left(k_{p}\right)$ of saturated rocks has been carried out by Geerstma and Smit [18] as,

$$
k_{p}=\frac{\Phi \eta}{2 \pi \rho f} \times \frac{1}{\sqrt{\frac{V_{\infty}^{4}-V_{p}^{2} V_{\infty}^{2}}{V_{p}^{2} V_{0}^{2}-V_{0}^{4}}}}
$$

where $\eta$ is fluid viscosity $\left(=1.0 \times 10^{-6} \mathrm{~m}^{2} / \mathrm{s}\right), \rho$ is fluid density $\left(=1.0 \times 10^{3} \mathrm{~kg} / \mathrm{m}^{3}\right)$, and $f$ is wave frequency. 


\section{Attenuation}

The earth layers as the medium of the propagation of elastic waves are getting attenuation effect resulted in the absorption of the energy by the layers. Seismic wave attenuation may be defined as a process of energy absorption by medium, which causes wave amplitude decreasing [19]. Attenuation depends on pressure, frequency, amplitude, temperature, porosity, fluid saturation, grain size, permeability, and volume of shale. Attenuation can also be expressed in terms of the quality factor $(Q)$, thus $Q$ becomes a standard to express how good of a rock may pass or impede the wave energy.

In many cases, attenuation $\left(Q^{-1}\right)$ is defined [20] as,

$$
\frac{1}{Q}=\frac{\delta}{\pi}=\frac{\alpha V}{\pi f}
$$

where $\delta$ is logarithmic decrement and $\alpha$ is attenuation coefficient. The combination of shale volume and varied porosity has been made by Klimentos and McCann [21] and it gives an equation that shows the correlation between attenuation coefficient $(\alpha)$, porosity $(\Phi)$, and shale volume $\left(C_{1}\right)$, as

$$
\alpha=0.0315 \phi+0.241 C_{1}-0.132
$$

\section{EXPERIMENTAL PROCEDURE}

This study has two different parts for calculating reservoir parameters and seismic parameters i.e. log data analysis and theoretically analysis. Log data analysis includes determining zone through qualitative analysis. This is needed to choose good reservoir rocks from a formation to be the prospect zone. The chosen reservoir rock from this KTP formation is sandstone. Shale volume should be determined first as a correction to the prospect zone through Eq. (20). Porosity can be determined by using Eq. (22) and then permeability may be obtained from Eq. (23). Attenuation data log analysis can be found by a combination of Eq. (29) and Eq. (30). To get P-wave velocity values, we should convert the value of sonic log unit $(\mu \mathrm{s} / \mathrm{ft})$ to the international unit $(\mathrm{m} / \mathrm{s})$. Theoretical analysis is the other way to determine reservoir parameters other than the conventional log data calculation. Table 3 shows the required calculation for each parameter theoretically.

The table also illustrates the possibility of determining other parameter values even if we have just P-wave velocity and porosity values, which are obtained from conventional log analysis. The calculation using Eq. (15) has been done by Sismanto and Susanna [22]. Some standard curves for difference porosity from Eq. (15) and Eq. (18) are shown in Fig. 1.

Table 3. The required parameters and its equation for theoretical analysis

\begin{tabular}{lcc}
\hline Parameter & units & equation \\
\hline P-wave velocity $\left(V_{P}\right)$ & $\mathrm{m} / \mathrm{s}$ & Log evaluated \\
S-wave velocity $\left(V_{S}\right)$ & $\mathrm{m} / \mathrm{s}$ & $(25)$ \\
Density $(\rho)$ & $\mathrm{gr} / \mathrm{m}^{3}$ & $(26)$ \\
Bulk modulus $(K)$ & $\mathrm{N} / \mathrm{m}^{2}$ & $(11)$ \\
Shear modulus $(\mu)$ & $\mathrm{N} / \mathrm{m}^{2}$ & $(9)$ \\
Poisson's ratio $(\sigma)$ & - & $(10)$ \\
Skeletal frame mod. $\left(K_{b}\right)$ & $\mathrm{N} / \mathrm{m}^{2}$ & $(7)$ \\
\hline Porosity $(\Phi)$ & $\%$ & Log evaluated \\
Biot's modulus $H$ & $\mathrm{~N} / \mathrm{m}^{2}$ & $(3)$ \\
Biot's modulus $M$ & $\mathrm{~N} / \mathrm{m}^{2}$ & $(5)$ \\
Biot's modulus $C$ & $\mathrm{~N} / \mathrm{m}^{2}$ & $(4)$ \\
Velocity in low frequency & $\mathrm{m} / \mathrm{s}$ & $(16)$ \\
$\left(V_{0}\right)$ & $\mathrm{m} / \mathrm{s}$ & $(17)$ \\
Velocity in high & $\mathrm{mD}$ & $(28)$ \\
frequency $\left(V_{\infty}\right)$ & - & $(15)$ and $(18)$ \\
\hline Permeability $\left(k_{p}\right)$ & & \\
Attenuation $\left(Q^{-1}\right)$ &
\end{tabular}




\section{RESULTS AND DISCUSSIONS}

Some standard curves from Eq. (15) and Eq. (18) have been constructed with the same parameter, but difference porosity value as shown in Fig. 1 (left and right) [22]. Fig. 1 to Fig. 5 indicate that the bigger the porosity, the lower the sensitivity of water saturation to attenuation, and the larger the water saturation, the higher the attenuation value would be had by a rock. From the figures, we can see that the standard curve form Eq. (18) is more sensitive than the standard curve from Eq. (15). The lower sensitivity especially occurs for porosity more than $30 \%$ and at the water saturation of more than $60 \%$. We see that the sensitivity difference looks like not so significant, but numerically it is very important in practice. However, in observing, it is rare for a number of rocks to have the porosity more than $60 \%$. In parallel, we are difficult to find the attenuation of rock so small like in the standard curves. Nevertheless, we can adjust the numerical value by calibration to the well, in particular for porosity or attenuation of seismic wave.

For construction and application these standard curves, the required parameters such as $\mathrm{P}$ wave velocity, porosity, permeability, and attenuation should have been calibrated. The calibration formulas for each parameter are obtained from the curves of each parameter obtained theoretically and log-analytically in Well Baretta. It is provided in Table 4.

Reservoir parameter values, obtained from those above formulas, then are used as inputs to Eq. (15) and Eq. (18) to construct a solution through standard curves. These standard curves make estimated water saturation values. The attenuation-water saturation relationship, Fig. 6 and Fig. 7 show an exponential curve which is constructed based on Turgut-Yamamoto formula Eq. (18) and Eq. (15). These curves for $4100 \mathrm{ft}$ to $4172 \mathrm{ft}$ with interval $4 \mathrm{ft}$ in Well Baretta show the larger the water saturation rate, the higher the attenuation value. The estimated water saturation values, identified through this standard curve, then named as crossplot - water saturation. The sensitivity of water saturation to the attenuation in standard curve Fig. 6 is better than Fig. 7. It means that the approximation by linearization of Eq. (15) make the relationship between attenuation to water saturation is significant.
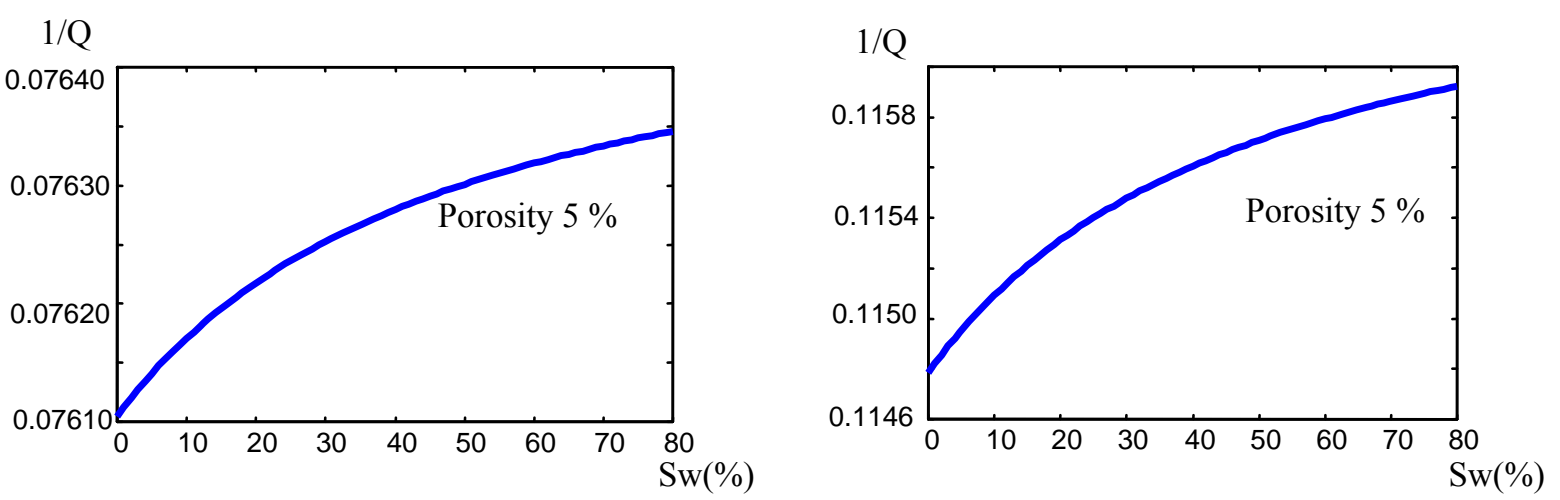

Fig. 1. Standard curves calculated of attenuation and water saturation from Eq. (15) (left) for 5\% porosity and Eq. (18) (right) in same others parameters.
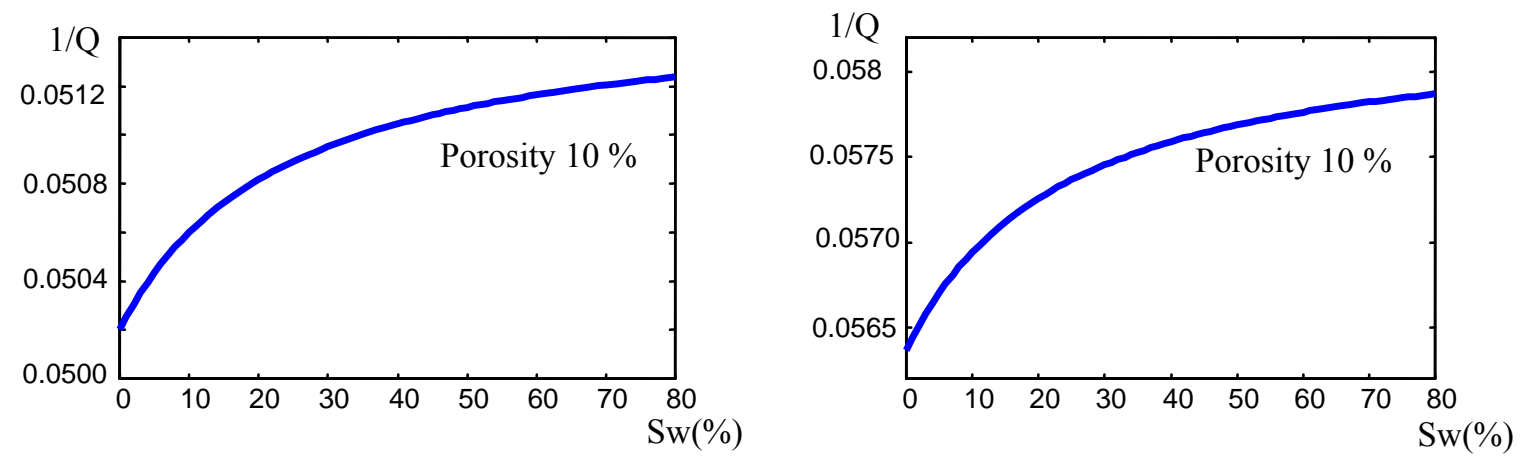

Fig. 2. Standard curves calculated attenuation and water saturation from Eq. (15) (left) for 10\% porosity and Eq. (18) (right) in same others parameters. 

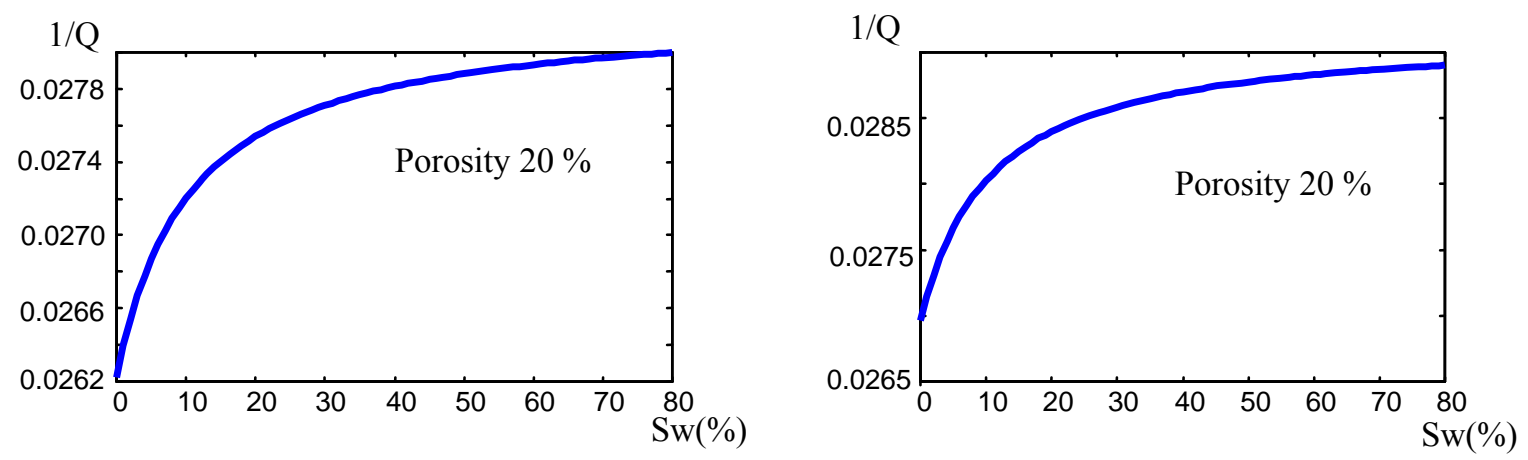

Fig. 3. Standard curves calculated attenuation and water saturation from Eq. (15) (left) for $20 \%$ porosity and Eq. (18) (right) in same others parameters.
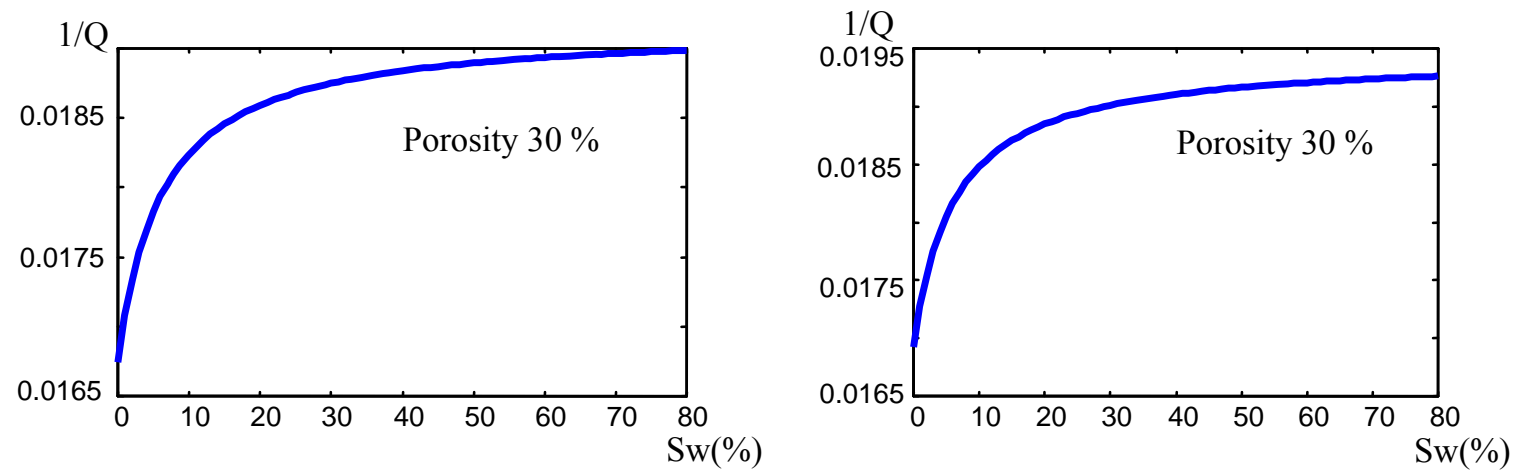

Fig. 4. Standard curves calculated attenuation and water saturation from Eq. (15) (left) for 30\% porosity and Eq. (18) (right) in same others parameters.
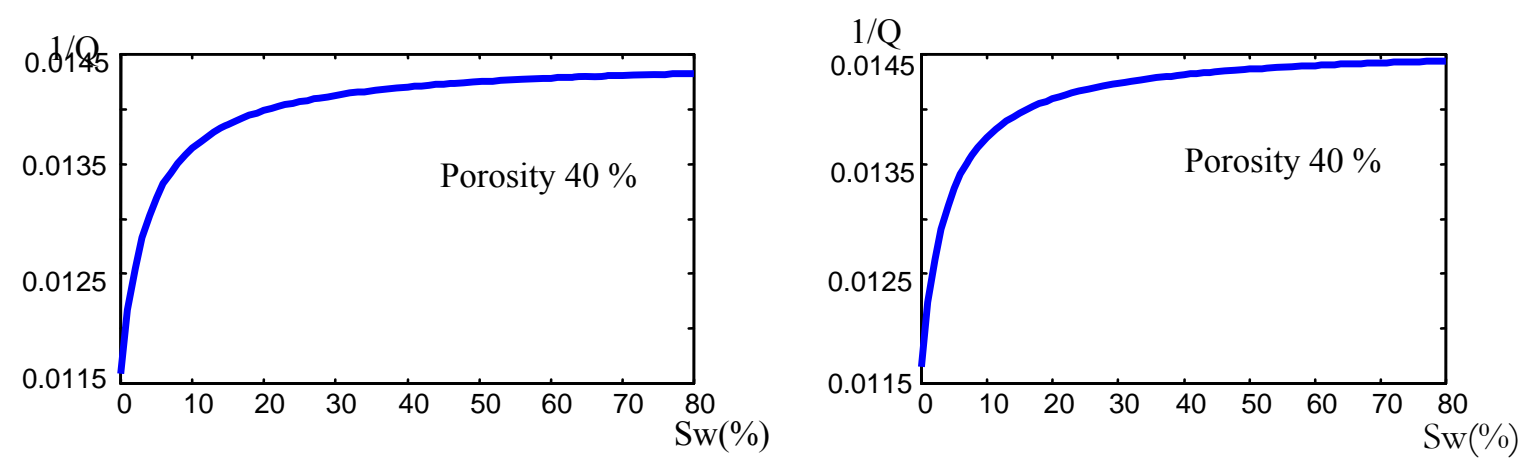

Fig. 5. Standard curves calculated attenuation and water saturation from Eq. (15) (left) for $40 \%$ porosity and Eq. (18) (right) in same others parameters.

Table 4. Calibration Eq. (18) and Eq. (15) for Reservoir Parameters of Baretta Well.

\begin{tabular}{|c|c|c|}
\hline Reservoir Paramete & Calibration Eq. (18) & Calibration Eq. (15) \\
\hline Porosity & $\emptyset_{\text {theory }}=\emptyset_{\text {log }}$ & $\varnothing_{\text {theory }}=\varnothing_{\text {log }}$ \\
\hline P-wave Velocity & $V p_{\text {theory }}=0.8971 V p_{\text {log }}-438.74$ & $V p_{\text {theory }}=0.8971 V p_{\text {log }}-438.74$ \\
\hline Permeability & $k_{p_{\text {theory }}}=3.4963 k_{p_{\text {log }}}+90.671$ & $k_{p_{\text {theory }}}=3.4963 k_{p_{\log }}+90.671$ \\
\hline Attenuation & $Q_{\text {theory }}^{-1}=-0.3328 Q_{\text {log }}^{-1}+0.046$ & $Q_{\text {theory }}^{-1}=-0.148 Q_{\text {log }}^{-1}+0.03$ \\
\hline
\end{tabular}




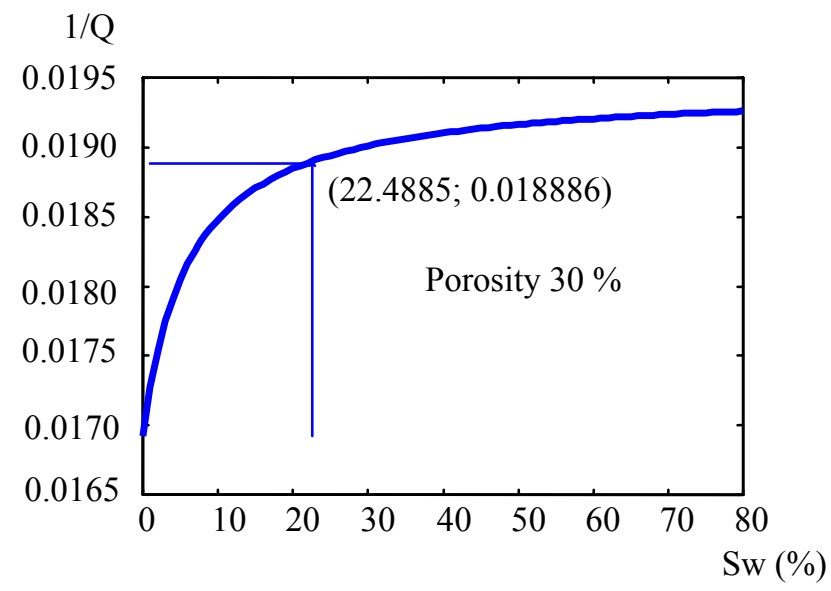

Fig. 6. Sample curve as the relationship of attenuation (1/Q) and water saturation (Sw) based on Turgut-Yamamoto formula Eq.(18) and the estimation.

The calibration formula for water saturation is found from the relationship curve of log-analytically water saturation and cross-plot water saturation, as is showed in Fig. 8. The calibration Eq.(18) for this formation is

and for Eq. (15) is

$$
S_{\text {wlog }}=0.961 S_{\text {wcrossplot }}+2.364
$$

$$
S_{\text {wlog }}=1.017 S_{\text {wcrossplot }}-0.767
$$

The products of water saturation calibration using Eq. (31) then are used to find calibrated water saturation. Fig. 8 shows various values of water saturation, which are obtained from different calculation i.e. by using conventional log evaluation, estimation formula (cross-plot), through Eq. (15) and Eq. (18), and it has the same trend.

It is also important to make other well testing. This is needed to know the error rate of other well test using the calibration formula of BARRETTA well. The well test, which is used in this study named as SG552 well. This well test is also located in the same field as BARRETTA well. The error, obtained from the calculation of conventional analysed and calibrated water saturation, is 3\%. This error may be different to other well.

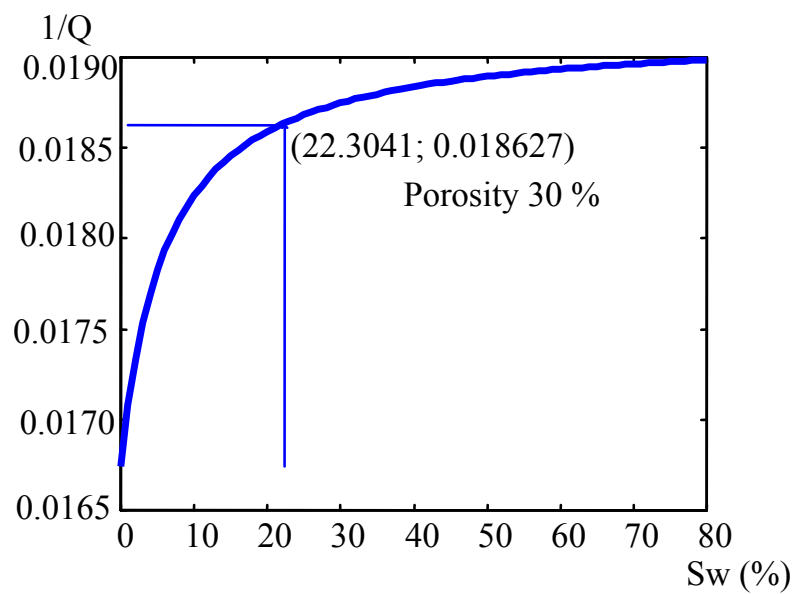

Fig. 7. Sample curve as the relationship of attenuation (1/Q) and water saturation (Sw) based on Biot-Turgut Yamamoto formula Eq. (15) and the estimation. 


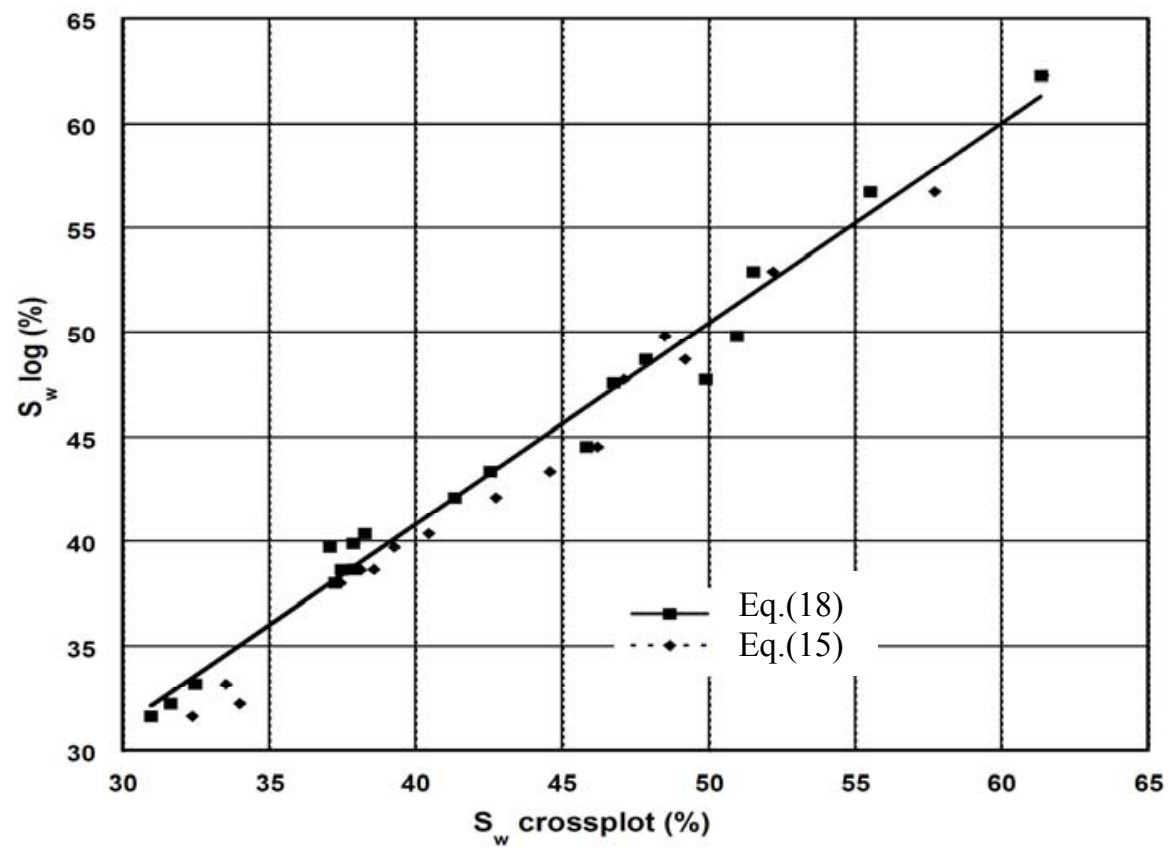

Fig. 8. The relationship curve of log-analytically water saturation and estimated water saturation to obtain calibration formula for water saturation for Eq.(18) and Eq.(15), and the interval data is in $4 \mathrm{ft}$.

\section{CONCLUSiON}

This study revised Turgut-Yamamoto formula to take advantage of the relationship of attenuation and water saturation of BARRETTA sandstone sample well from a North Sumatera Basin. The attenuation increases proportionally to the increasing water saturation after linearization of Turgut-Yamamoto curve. The linear relationship between attenuation and water saturation is more obvious than the original of Turgut-Yamamoto curve so that it is easier to determine water saturation parameter via cross-plot. Based on the linearization of Turgut-Yamamoto formula, the method could be considered to determine water saturation of a well, with its error of $3 \%$.

\section{REFERENCES}

[1] C. McCann, and D.M. McCann, A Theory of compressional wave attenuation in noncohesive sediments. Geophysics, 50, 1311-1317. 1985.

[2] F. Rafavich, Kendall, C.H.St.C. and Todd, T.P. The relationship between acoustic properties and the petrographic character of carbonate rocks. Geophysics, 49, 1632-1636. 1984.

[3] A.I. Best, and M.S. Sams, Compressional wave velocity and attenuation at ultrasonic and sonic frequencies in near-surface sedimentary rocks. Geophysical Prospecting, 45, 327-344. 1997.

[4] G. Asquith, and D. Krygowski, Basic Well Log Analysis, 2nd Edition, The American Association of Petroleum Geologist, Tulsa, Oklahoma. 2004.

[5] A.I. Best, C. McCann, and J. Sothcott, The Relationships Between The Velocities, Attenuations and Petrophysical Properties of Reservoir Sedimentary Rocks, Geophysical Prospecting, 42, 151-178. 1994.

[6] S.I. Purwagandhi and. Sismanto, Estimation of Reservoir Permeability from Sismanto's Equation in 2D Seismic Data of Teapot Dome Field. 77th EAGE Conference and Exhibition 2015, IFEMA Madrid, Spain, 1-4 June 2015.

[7] Sismanto, Rock physics: Approximation of permeability estimation and water saturation based on seismic data. Graha Ilmu, Yogyakarta. 2013. (In Bahasa Indonesia).

[8] M. A. Biot, Theory of Propagation of Elastic Waves in A Fluid Saturated Porous Solid I. Low-Frequency Range, J. Acoust. Soc. Am., 28, 168-178. 1956.

[9] M. A. Biot, Theory of Propagation of Elastic Waves in A Fluid Saturated Porous Solid II. Higher Frequency Range, J. Acoust. Soc. Am., 28, 179-191. 1956.

[10] A. Turgut, and T. Yamamoto, Measurement of acoustic wave velocities and attenuation in marine sediments, J. Acoust. Soc. Am., 87, 2376-2383. 1990.

[11] R.D. Stoll, Acoustic waves in saturated sediments, Physics of sound in marine sediments, Plenum Press, p.19-39. 1974.

[12] A. Turgut, and T. Yamamoto, Synthetic seismograms for marine sediments and determination of porosity and permeability. Geophysics, 53, 1056-1067. 1988.

[13] Sismanto, 2016. A New Approximation of Water Saturation Estimation Based on Vertical Seismic Profiling Data. Int. Journal of Engineering Research and Applications. Vol. 6, Issue 1, pp.49-52, (Part - 4) January 2016,

[14] D.W. Hilchie, Applied Openhole Log Interpretation, Golden, Colorado, D.W. Hilchie, Inc., p.309. 1978

[15] V.V. Larionov, Borehole Radiometry, Moscow, U.S.S.R., Nedra. 1969.

[16] J.P Castagna,., M.L. Batzle, and T.K. Kan, Rock physics the link between rock properties and AVO response, in Offset-Dependent Reflectivity-Theory and Practice of AVO Analysis, Geophysics, 8, 3-36. 1993.

[17] G. Mavko, T. Mukerji, and J. Dvorkin, The Rock Physics Handbook: Tool for Seismic Analysis in Porous Media, Cambridge Univ. Press, USA, 1998.

[18] I. Geertsman, and D.C. Smit, Some aspects of elastic wave propagation in fluid-saturated porous solids. Geophysics, 26, 161-181.1961.

[19] Munadi, S, Aspek Fisis Seismologi Eksplorasi, FMIPA, Universitas Indonesia, Indonesia, p93-102, 2000. 
[20] D.H Johnston, and M.N. Toksöz, Seismic Wave Attenuation, SEG Geophysics Reprint series No.2.

[21] T.Klimentos, and C. McCann, Relationships among compressional wave attenuation, porosity, clay content and permeability in sandstone, Geophysics, 55, 998-1014. 1990.

[22] Sismanto and R. E. Susanna, Estimation of Water Saturation Using Crossplot Method Based on Attenuation Analysis of Biot-TurgutYamamoto. Proceeding of International Conference of Mathematical and Natural Science 2008, Bandung 28-30 October 2008, Bandung, Indonesia.

\section{AUTHOR PROFILE}

Sismanto received his undergraduate in physics from Gadjah Mada University Indonesia in 1985, and master degree (1995) and PhD (2003) from ITB (Institute Teknologi Bandung) Indonesia in Geophysics. His research interests are in the rock physics, Exploration Geophysics, and Hazards. Now he is one of the lecturer in Geophysics Study Program of Gadjah Mada University, Indonesia.

Rah Ajeng Putri received her undergraduate in physics from Gadjah Mada University Indonesia in 2008. Now she is a Senior Geophysicist on SpectrumGeo Multi Client and Seismic Imaging. 